\title{
Stronger Self-Focusing of Hermite-Cosh-Gaussian (HChG) Laser Beam in Plasma
}

\author{
Niti Kant*, Vikas Nanda \\ Department of Physics, Lovely Professional University, Phagwara, India \\ Email: ${ }^{*}$ nitikant@yahoo.com
}

Received 25 May 2014; revised 8 July 2014; accepted 18 August 2014

Copyright (C) 2014 by authors and OALib.

This work is licensed under the Creative Commons Attribution International License (CC BY). http://creativecommons.org/licenses/by/4.0/

(c) †) Open Access

\section{Abstract}

The relativistic self-focusing of HChG beam in the plasma has been investigated theoretically using WKB and paraxial ray approximation for mode indices 0,1 and 2 for different values of pulse amplitude, normalized plasma density and decentred parameter. The plot between beam width parameter $f$ and the normalized length $\xi$ for different value of normalized density has been reported and indicates the enhancement of the self-focusing phenomena of the laser beams in the plasmas. Also the focusing and defocusing of HChG beam in the plasma having dependency on decentred parameter and frequency of incident laser beam is reported.

\section{Keywords}

Self-Focusing, Plasma Density, Nonlinearity, Hermite-Cosh-Gaussian Beam

Subject Areas: Plasma, Theoretical Physics

\section{Introduction}

The interaction of light with matter is one of the basic phenomena in nature. Moreover the advancement in the short pulse laser technology has enabled experiments using laser pulses focused on extremely high intensity of the order of $10^{20} \mathrm{~W} / \mathrm{cm}^{2}$. Self-focusing of laser beams in plasmas [1]-[5] becomes one of the most interesting and fascinating field of research for several decades due to its various applications like the generation of inertial fusion energy driven by lasers [6], optical harmonic generation [7], the production of quasi mono-energetic electron bunches [8], laser driven fusion [9], x-ray lasers and the laser driven accelerators [10]. These applications need the laser pulse to propagate over several Rayleigh lengths in the plasmas without loss of energy.

The dielectric constant of plasma modifies greatly with the increase in intensity of the laser beam and it leads

*Corresponding author. 
to self-focusing of the beam. The variation in the electron density in plasmas is caused by the propagating laser pulse of extremely high intensities of the order ranging from $10^{17}-10^{20} \mathrm{~W} / \mathrm{cm}^{2}$. Such high intensity laser pulses provide sufficient energy to the constituent particles of the plasmas which suddenly increase the speed of electrons nearly equal to the speed of light enhancing the mass of the electron and hence give rise to the relativistic effect [2]. Relativistic self-focusing and self-channeling of Gaussian laser beam has been, recently, reported by Singh et al. [5] by applying moment theory approach to solve the non-linear differential equation for beam width parameter and then solve it numerically by Runge-Kutta method.

Recently, theoretical investigators have focused their attention on paraxial wave family of laser beams. Hermite-cosh- Gaussian beam is one of the solutions of paraxial wave equation and such HChG beam can be obtained in the laboratory by the superposition of two decentred Hermite-Gaussian beams as Cosh-Gaussian ones. Propagation of HChG beams in plasmas has been studied theoretically earlier by Belafhal et al. [3] and Patil et al. [11]. The focusing of HChG laser beams in magneto-plasma by considering ponderomotive nonlinearity has been theoretically examined by Patil et al. [12] and they reported the effect of mode index and decentered parameter on the self-focusing of the beams.

The present work is dedicated to the study of the relativistic self-focusing of HChG beam in plasma. We derive the equations for beam width parameter for HChG beam and solve them numerically by applying WentzelKramers-Brillouin (WKB) approximation and Paraxial approximation [13] [14] for mode index 0, 1 and 2 and observe the enhancement in the self-focusing of the laser beams.

\section{Nonlinear Dynamics}

The field distribution of Hermite-Cosh-Gaussian (HChG) laser beams propagating in plasma along $z$-axis is of the following form:

$$
E(r, z)=\frac{E_{0}}{f(z)}\left[H_{m}\left(\frac{\sqrt{2} r}{r_{0} f(z)}\right)\right] \mathrm{e}^{\frac{b^{2}}{4}}\left\{\mathrm{e}^{-\left(\frac{r}{r_{0} f(z)}+\frac{b}{2}\right)^{2}}+\mathrm{e}^{-\left(\frac{r}{r_{0} f(z)}+\frac{b}{2}\right)^{2}}\right\}
$$

where $E_{0}$ is the amplitude of HChG laser beam for the central position at $r=z=0$, " $f(z)$ " is the dimensionless beam width parameters, " $H_{m}$ " is the Hermite polynomial of $m^{\text {th }}$ order, " $r_{0}$ " is the spot size of the beam and " $b$ " is the decentered parameter of the beam.

The dielectric constant for the non-linear medium (collision-less plasma) is of the following form:

$$
\varepsilon=\varepsilon_{0}+\phi\left(E E^{*}\right)
$$

where $\varepsilon_{0}=1-\omega_{P 0}^{2} / \gamma \omega^{2}, m=m_{0} \gamma, \gamma=1 / \sqrt{1-v^{2} / c^{2}}$ and $\omega_{P 0}=\left(4 \pi e^{2} n_{0} / m\right)^{1 / 2}$ is the plasma frequency at equilibrium. Here " $\varepsilon_{0}$ " and " $\emptyset$ " represent the linear and non-linear parts of the dielectric constant respectively, $m_{0}$, $\& \omega$ are the electronic charge, rest mass of the electron and frequency of the incident laser respectively and " $n_{0}$ " is the equilibrium electron density.

The non-linearity induced due to ponderomotive forces in the dielectric constant in collisionless plasma and hence non-linear part of the dielectric constant can be written as [15]

$$
\phi\left(E E^{*}\right)=\left(\frac{\omega_{P 0}^{2}}{\gamma \omega^{2}}\right)\left[1-\exp \left(-\frac{3 m_{0} \gamma \alpha_{1} E E^{*}}{4 M}\right)\right]
$$

where $\alpha_{1}=e^{2} M / 6 m_{0}^{2} \gamma^{2} \omega^{2} k_{B} T_{0}$, here " $M$ " is the mass of the scatterer in the plasma, " $K_{B}$ " is the Boltzmann constant and " $T_{0}$ " is the plasma temperature at equilibrium.

\section{Self-Focusing Equations}

Wave equation can be deduced by using Maxwell's equations and can be written as

$$
\nabla^{2} \boldsymbol{E}-\frac{\varepsilon}{c^{2}} \cdot \frac{\partial^{2} \boldsymbol{E}}{\partial t^{2}}+\nabla\left(\boldsymbol{E} \frac{\nabla(\varepsilon)}{\varepsilon}\right)=0
$$

Consider a plane polarized wave with electric field vector given by 


$$
\boldsymbol{E}=\boldsymbol{A}(r, z) \exp i(\omega t-k z)
$$

where $A(r, z)$ is the complex amplitude of the electric field. Thus,

$$
\partial^{2} \boldsymbol{E} / \partial t^{2}=-\omega^{2} \boldsymbol{E}
$$

Hence Equation (4) becomes

$$
\nabla^{2} \boldsymbol{E}+k^{2} \boldsymbol{E}+\nabla\left(\boldsymbol{E} \frac{\nabla(\varepsilon)}{\varepsilon}\right)=0
$$

where $k=(\omega / c) \varepsilon^{1 / 2}$. For $\left(1 / k^{2}\right) \nabla^{2}(\ln \varepsilon) \ll 1$.

$$
\frac{\partial^{2} E}{\partial z^{2}}+\frac{\partial^{2} E}{\partial r^{2}}-\frac{1}{r} \frac{\partial E}{\partial r}+\frac{\varepsilon \omega^{2}}{c^{2}} \boldsymbol{E}=0
$$

We know that the solution of Equation (8) is of the form,

$$
\boldsymbol{E}=\boldsymbol{A}(r, z) \exp i\left(\omega t-\frac{z}{c} \sqrt{\omega^{2}-\frac{\omega_{p 0}^{2}}{\gamma}}\right)
$$

Substituting this value in Equation (8), we get

$$
2 i k \frac{\partial A}{\partial z}=\frac{\partial^{2} A}{\partial r^{2}}+\frac{1}{r} \frac{\partial A}{\partial r}+\frac{k^{2} A}{\varepsilon_{0}} \phi\left(A A^{*}\right)
$$

The solution of Equation (10) is of the form,

$$
A(r, z)=A_{0 p}(r, z) \exp [-i k S(r, z)]
$$

Here " $A_{0 p}$ " and " $S$ " are the real function of $r$ and $z$.

Using Equation (9) in Equation (8) and separating real and imaginary parts, we get Real part is

$$
2 \frac{\partial S}{\partial z}+\left(\frac{\partial S}{\partial r}\right)^{2}=\frac{1}{k^{2} A_{0 p}}\left(\frac{\partial^{2} A_{0 p}}{\partial r^{2}}+\frac{1}{r} \frac{\partial A_{0 p}}{\partial r}\right)+\frac{\phi\left(A_{0 p}^{2}\right)}{\varepsilon_{0}}=0
$$

Imaginary part is

$$
2 \frac{\partial A_{0 p}}{\partial z}+\frac{1}{r} A_{0 p} \frac{\partial S}{\partial r}+A_{0 p} \frac{\partial^{2} S}{\partial r^{2}}+2 \frac{\partial A_{0 p}}{\partial r} \frac{\partial S}{\partial r}=0 .
$$

The solution of Equations (12) \& (13) are of the form,

$$
A_{0 p}^{2}=\frac{E_{0}^{2}}{f^{2}(z)}\left[H_{m}\left(\frac{\sqrt{2} r}{r_{0} f}\right)\right] \mathrm{e}^{\frac{b^{2}}{2}}\left\{\mathrm{e}^{-2\left(\frac{r}{r_{0} f}+\frac{b}{2}\right)^{2}}+\mathrm{e}^{-2\left(\frac{r}{r_{0} f}-\frac{b}{2}\right)^{2}}+2 \mathrm{e}^{-\left(\frac{2 r^{2}}{r_{0}^{2} f^{2}}+\frac{b^{2}}{2}\right)}\right\}
$$

And

$$
S=\frac{r^{2}}{2} \beta(z)+\phi(z)
$$

with $\beta(z)=(1 / f) \partial f / \partial z$, represents the inverse of the radius of curvature of the wavefront of the laser beam. Here $\phi(z)$ is an arbitrary function of " $z$ ". Using these values in Equation (12), we get,

For $\boldsymbol{m}=\mathbf{0}$

$$
\frac{\partial^{2} f}{\partial \xi^{2}}=\frac{1}{f^{3}}\left[4-2 b^{2}-12 b^{4}+2 b^{6}-\frac{6 \alpha E_{0}^{2} r_{0}^{2} m_{0} \omega_{p 0}^{2}}{\gamma^{2} c^{2} M} \mathrm{e}^{\frac{b^{2}}{2}} \mathrm{e}^{\frac{-3 \alpha E_{0}^{2} m_{0}}{M \gamma f^{2}}}\right]
$$


For $m=1$

$$
\frac{\partial^{2} f}{\partial \xi^{2}}=\frac{1}{f^{3}}\left[4+2 b^{2}-20 b^{4}+2 b^{6}-\frac{12 \alpha E_{0}^{2} r_{0}^{2} m_{0} \omega_{p 0}^{2}}{\gamma^{2} c^{2} M} \mathrm{e}^{\frac{b^{2}}{2}}\left(b^{2}-2\right)\right]
$$

For $\boldsymbol{m}=2$

$$
\frac{\partial^{2} f}{\partial \xi^{2}}=\frac{1}{f^{3}}\left[-28+78 b^{2}-4 b^{4}+22 b^{6}+\frac{24 \alpha E_{0}^{2} r_{0}^{2} m_{0} \omega_{p 0}^{2}}{\gamma^{2} c^{2} M} \mathrm{e}^{\frac{b^{2}}{2}} \mathrm{e}^{\frac{-12 \alpha E_{0}^{2} m_{0}}{M \gamma f^{2}}}\left(5-2 b^{2}\right)\right]
$$

where beam width parameter $f$ is a function of $\xi$, here $\xi=z / R_{d}$ and $R_{d}$ is the diffraction length. Similarly Equation (14) gives the condition, $\partial f / \partial z=0, f=$ constant.

\section{Results and Discussion}

The variation of beam width parameter $f$ with normalized propagation distance $\xi$ for different values of relative plasma frequency; $\omega_{p 0} / \omega=0.7,0.8$ and 0.9 has been seen for different parameters given as $b=0.67, r_{0}=$ $3 \times 10^{-6} \mathrm{~m}, \omega=9.4 \times 10^{14} \mathrm{rad} / \mathrm{s}$ and $\alpha E_{0}^{2}=0.3$. From Figure 1 , it is clear that with the increase in plasma density, the beam width parameter $f$ decreases greatly. Hence self-focusing of laser beam becomes more and more strong which supports the results of Sadighi-Bonabi et al. [8]. The results obtained are also in good agreement with the results reported by Singh et al. [5]. In Figure 2, the variation of beam width parameter $f$ with dimensionless normalized propagation distance $(\xi)$ for different values of relative plasma density; $\left(\omega_{p 0} / \omega\right)=0.5,0.6$ and 0.7 has been observed. The plot obtained clarifies that for $\alpha E_{0}^{2}=0.2$ and $\gamma=0.99$, beam width parameter decreases with the increase in relative plasma frequency $\left(\omega_{p 0} / \omega\right)$. Hence self-focusing of laser beam becomes stronger and earlier as well. Also for higher values of relative plasma density, self-focusing phenomenon occurs earlier with the normalized propagation distance. The results obtained are in good agreement with the results reported in ref. [5].

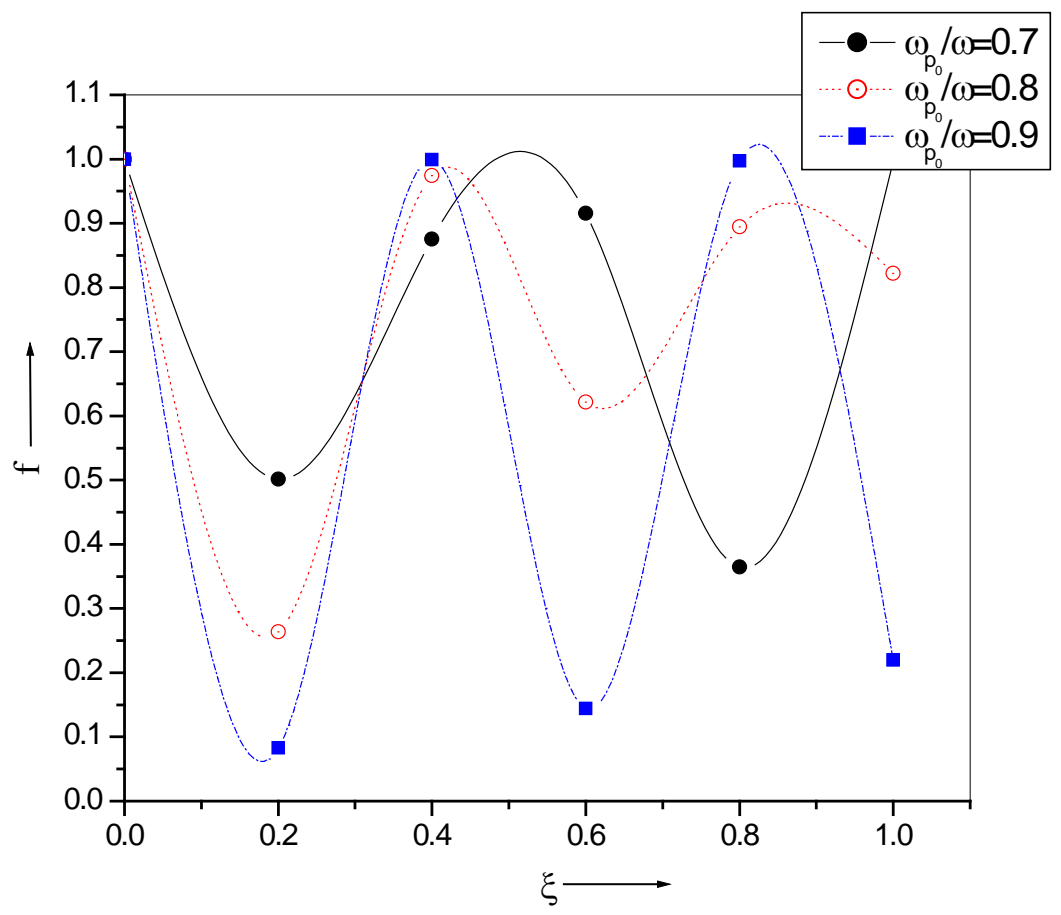

Figure 1. Variation of beam width parameter $(f)$ with normalized propagation distance $\xi$ for relative density $\omega_{p 0} / \omega=0.7$ (Black curve) $\omega_{p 0} / \omega=0.8$ (Red curve) and $\omega_{p 0} / \omega=0.9$ (Blue curve) for various parameter taken as $m=0, b=0.67, r_{0}=3 \times 10^{-6} \mathrm{~m}, \omega=9.4 \times 10^{14} \mathrm{rad} / \mathrm{s}$ and $\alpha E_{0}^{2}=0.3$. 


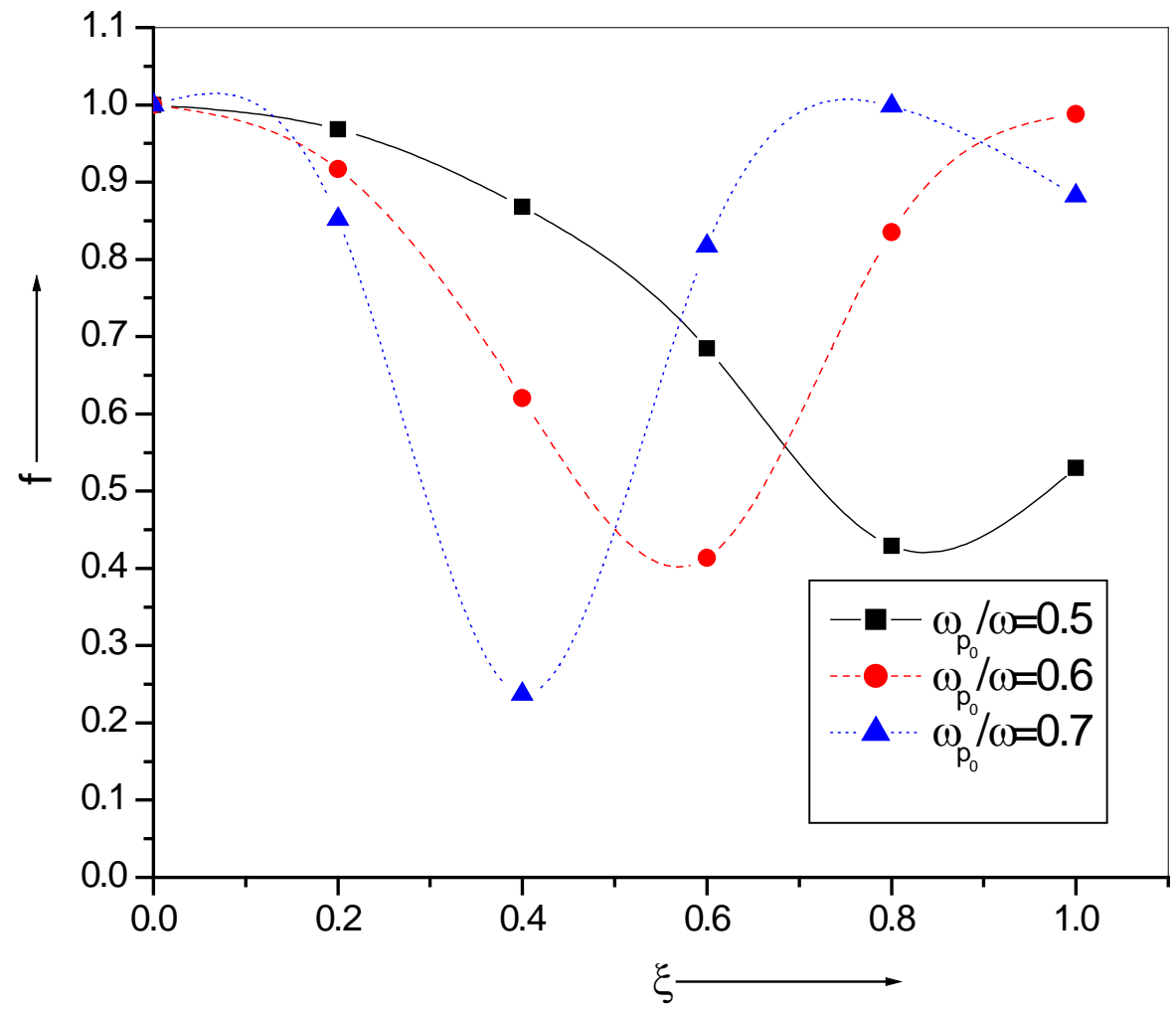

Figure 2. Variation of beam width parameter $f$ with normalized distance $\xi$ for $\omega_{p 0} / \omega=$ 0.5 (Black curve) $\omega_{p 0} / \omega=0.6$ (Red curve) $\omega_{p 0} / \omega=0.7$ (Blue curve) for parameters $\gamma=$ 0.99, $m=0, b=0, r_{0}=3 \times 10^{-5} \mathrm{~m}, \omega=3.5 \times 10^{14} \mathrm{rad} / \mathrm{s}$ and $\alpha E_{0}^{2}=0.2$.

Figure 3 represents the variation of beam width parameter $f$ with dimensionless normalized propagation distance $\xi$, for mode index $m=1$, at different values of frequency of incident laser beam viz. $\omega=9.3 \times 10^{14} \mathrm{rad} / \mathrm{s}$ (Black curve) $\omega=9.4 \times 10^{14} \mathrm{rad} / \mathrm{s}$ (Red curve) $\omega=9.5 \times 10^{14} \mathrm{rad} / \mathrm{s}$ (Blue curve) for considering various parameters as $b=1, r_{0}=3 \times 10^{-6} \mathrm{~m}$, relative mass $\left(m_{0} / M\right)=0.5$ and $\alpha E_{0}^{2}=0.1$. From Figure 3, one can easily conclude that for $\omega=9.3 \times 10^{14} \mathrm{rad} / \mathrm{s}$ (Black curve), strong focusing of laser beam occurs and for $\omega=9.4 \times 10^{14}$ $\mathrm{rad} / \mathrm{s}$ (Red curve) and $\omega=9.5 \times 10^{14} \mathrm{rad} / \mathrm{s}$ (Blue curve), laser beam gets defocused for decentered parameter $b=$ 1. It happens because the increase in the value of frequency of incident beam varies the density of the plasma greatly. Hence focusing and de-focusing nature of HChG beam for mode index $m=1$ is decided by the choice of the frequency of the laser beam propagating through the plasma medium. Patil et al. [16] investigate analytically HChG laser beam propagation in non-degenerate germanium having space charge neutrality for first three mode indices $(m)$ and conclude that decentered parameter $(b)$ changes the nature of self focusing/de-focusing significantly. In addition to that we conclude that frequency of incident laser beam $(\omega)$ give additional freedom to choose a parameter which can control the nature of self-focusing/de-focusing of HChG laser beam for mode indices $m=1$. These results are also in agreement with the results obtained by Kant $e t$ al. [17].

Figure 4, represents the variation of beam width parameter $(f)$ with normalized propagation distance $(\xi)$ for mode index $m=2$ for decentered parameter $b=0,1$ and 2 with $\omega=9.4 \times 10^{14} \mathrm{rad} / \mathrm{s}, r_{0}=3 \times 10^{-6} \mathrm{~m}, \omega_{p 0} / \omega=0.5$, $m_{0} / M=0.25$ and $\alpha E_{0}^{2}=0.1$ For decentered parameter $b=0$, self-focusing of the HChG beam occurs and for decentered parameter $b=1$ and 2, defocusing of the HChG beam take place. The second non-linear term of Equation (17) dominates over the first term for $b=0$ and cause the self-focusing of HChG beam $(m=2)$. For $b=0$ and 1, the beams propagates without focusing or defocusing nearly up to $\xi=0.5$ and then a small effect of self-focusing is observed. The results obtained are in agreement with the results reported by Patil et al., [11] in case of propagation of Hermite-Cosh-Gaussian laser beams in n-InSb. Various studies have been done to achieve stronger self-focusing by using various laser profiles [18]-[22]. 


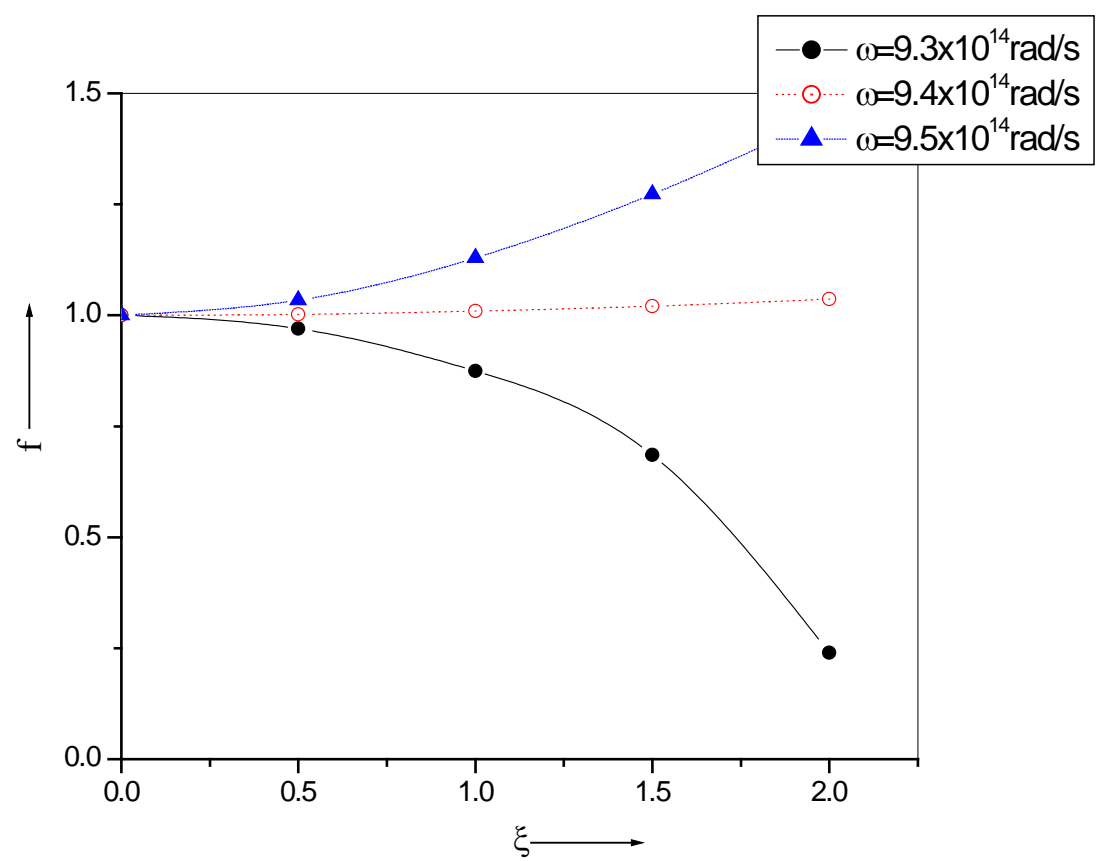

Figure 3. Variation of beam width parameter $f$ with normalized propagation distance $\xi$ for propagating laser frequency $\omega=9.3 \times 10^{14} \mathrm{rad} / \mathrm{s}$ (Black curve) $\omega=9.4 \times 10^{14} \mathrm{rad} / \mathrm{s}$ (Red curve) $\omega=9.5 \times 10^{14} \mathrm{rad} / \mathrm{s}$ (Blue curve) for parameter $m=1, b=1 r_{0}=3 \times 10^{-6} \mathrm{~m}$, relative mass $\left(m_{0} / M\right)=0.5$ and $\alpha E_{0}^{2}=0.1$.

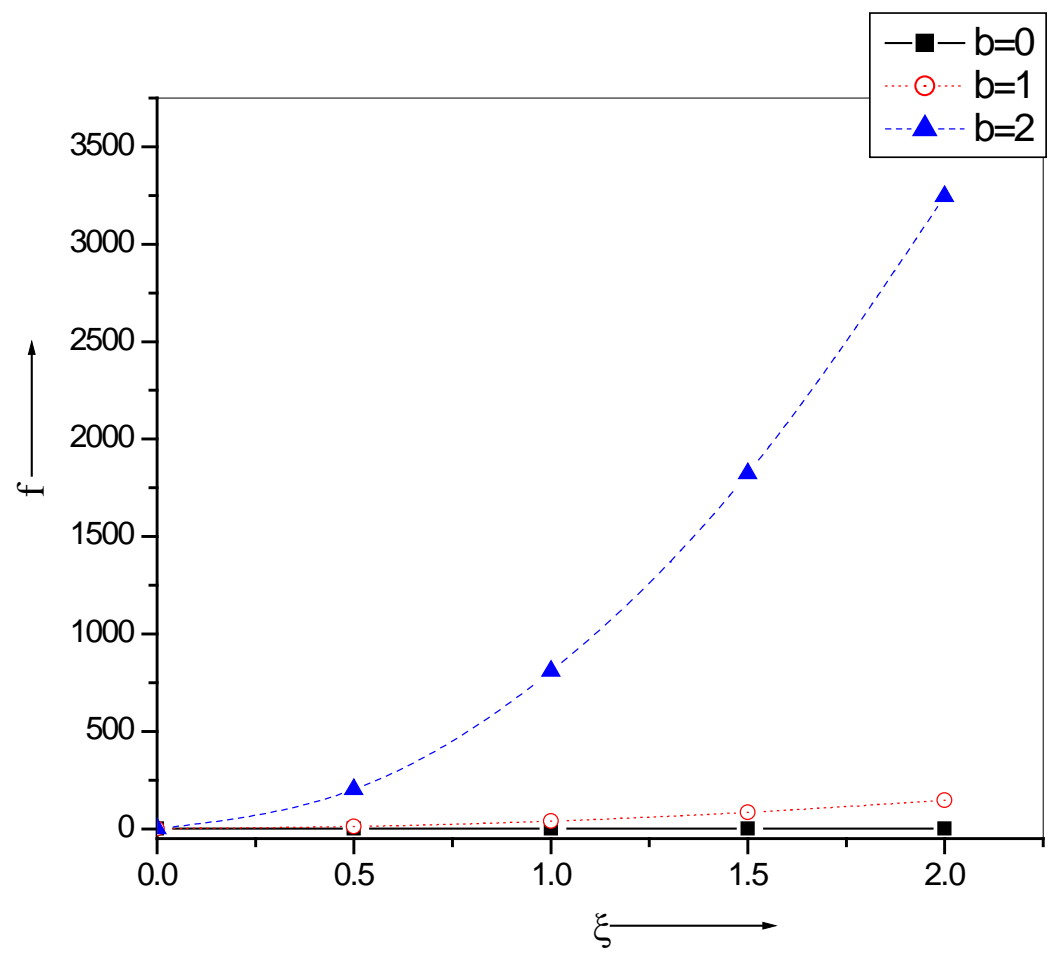

Figure 4. Variation of beam width parameter $f$ with normalized propagation distance $\xi$ for mode index $m=2$ for decentered parameter $b=0$ (Black curve), $b=1$ (Red curve) and $b=2$ (Blue curve) for parameter $\omega=9.4 \times 10^{14} \mathrm{rad} / \mathrm{s}, r_{0}=3 \times 10^{-6} \mathrm{~m}, \omega_{p 0} / \omega=0.5, m_{0} / M=0.25$ and $\alpha E_{0}^{2}=0.1$. 


\section{Conclusion}

From the above results, we conclude that focusing/defocusing phenomena of HChG beams for mode indices $m=$ 0,1 and 2, will be controlled with the frequency of the laser beam propagating through the plasma and decentered parameter. Moreover, self-focusing becomes stronger and earlier with the increase in plasma density for mode index $m=0$. For $m=2$, Hermite-Cosh-Gaussian beam exhibits self-focusing nearly up to normalized propagation distance $\xi=0.5$ for decentered parameter $b=0$ and 1 while for higher values of decentered parameter i.e. $b=2$, purely defocusing of the beam occurs. The results obtained in this study adds some more choices of parameters viz. mode indices $(m)$, decentered parameter $(b)$ and frequency of incident laser beam $(\omega)$ for investigators working in the field of self-focusing of laser beams of various profiles. Present study may be useful for the scientist working on laser induced fusion.

\section{Acknowledgements}

This work was supported by a financial grant from CSIR, New Delhi, India, under Project No. 03(1277)/ 13/EMR-II.

\section{References}

[1] Hora, H. (1969) Self-Focusing of Laser Beams in a Plasma by Ponderomotive Forces. Zeitschrift für Physik, 226, 156159. http://dx.doi.org/10.1007/BF01392018

[2] Siegrist, M.R. (1976) Self-focusing in a Plasma Due to Ponderomotive Forces and Relativistic Effects. Optics Communications, 16, 402-407.

[3] Belafhal, A. and Ibnchaikh, M. (2000) Propagation Properties of Hermite-Cosh-Gaussian Laser Beams. Optics Communications, 186, 269-276. http://dx.doi.org/10.1016/S0030-4018(00)01081-6

[4] Hafizi, B., Ting, A., Sprangle, P. and Hubbard, R.F. (2000) Relativistic Focusing and Ponderomotive Channeling of Intense Laser Beams. Physical Review E, 62, 4120-4125.

[5] Singh, A. and Walia, K. (2010) Relativistic Self-Focusing and Self-Channeling of Gaussian Laser Beamin Plasma. Applied Physics B, 101, 617-622. http://dx.doi.org/10.1007/s00340-010-4230-4

[6] Yazdani, E., Cang, Y., Sadighi-Bonabi, R., Hora, H. and Osman, F. (2009) Layers from Initial Rayleigh Density Profile by Directed Nonlinear Force Driven Plasma Blocks for Alternative Fast Ignition. Laser and Particle Beams, 27, 149156. http://dx.doi.org/10.1017/S0263034609000214

[7] Banerjee, S., Valenzuela, A.R., Shah, R.C., Maksimchuk, A. and Umstadter, D. (2002) High Harmonic Generation in Relativistic Laser Plasma Interaction. Physics of Plasmas, 9, 2393-2398. http://dx.doi.org/10.1063/1.1470167

[8] Sadighi-Bonabi, R., Navid, H.A. and Zobdeh, P. (2009) Observation of Quasi Mono-Energetic Electron Bunches in the New Ellipsoid Cavity Model. Laser and Particle Beams, 27, 223-231. http://dx.doi.org/10.1017/S0263034609000299

[9] Brueckner, K.A., Jorna, S. (1974) Laser Driven Fusion. Reviews of Modern Physics, 46, 325-367. http://dx.doi.org/10.1103/RevModPhys.46.325

[10] Faure, J., Glinec, Y., Pukhov, A., Kiselev, S., Gordienko, S., Lefebvre, E., Rousseau, J.P., Burgy, F. and Malka, V. (2004) A Laser Plasma Accelerator Producing Mono Energetic Electron Beams. Nature, 431, 541-544. http://dx.doi.org/10.1038/nature02963

[11] Patil, S.D., Takale, M.V. and Dongare, M.B. (2008) Propagation of Hermite-Cosh-Gaussian Laser Beams in n-InSb. Optics Communications, 281, 4776-4779. http://dx.doi.org/10.1016/j.optcom.2008.05.045

[12] Patil, S.D., Takale, M.V., Navare, S.T. and Dongare, M.B. (2010) Focusing of Hermite-Cosh-Gaussian Laser Beams in Collisionless Magnetoplasma. Laser and Particle Beams, 28, 343-349. http://dx.doi.org/10.1017/S0263034610000297

[13] Akhmanov, S.A., Sukhorukov, A.P. and Khokhlov, R.V. (1968) Self-Focusing and Diffraction of Light in a Nonlinear Medium. Soviet Physics Uspekhi, 10, 609-636. http://dx.doi.org/10.1070/pu1968v010n05abeh005849

[14] Kant, N., Gupta, D.N. and Suk, H. (2011) Generation of Second Harmonic Radiations of a Self-Focusing Laser from a Plasma with Density Transition. Physics Letters A, 375, 3134-3137. http://dx.doi.org/10.1016/j.physleta.2011.06.062

[15] Sodha, M.S., Ghatak, A.K. and Tripathi, V.K. (1974) Self-Focusing of Laser Beams. Tata-McGraw-Hill, New Delhi.

[16] Patil, S., Takale, M., Fulari, V. and Dongare, M. (2008) Propagation of Hermite-Cosh-Gaussian Laser Beams in NonDegenerate Germanium Having Space Charge Neutrality. Journal of Modern Optics, 55, 3529-3535.

[17] Kant, N., Saralch, S. and Singh, H. (2011) Ponderomotive Self-Focusing of a Short Laser Pulse under a Plasma Density Ramp. Nukleonika, 56, 149-153. 
[18] Nanda, V. and Kant, N. (2014) Strong Self-Focusing of a Cosh-Gaussian Laser Beam in Collisionless Magneto-Plasma under Plasma Density Ramp. Physics of Plasmas, 21, Article ID: 072111. http://dx.doi.org/10.1063/1.4889862

[19] Nanda, V. and Kant, N. (2014) Enhanced Relativistic Self-Focusing of Hermite-Cosh-Gaussian (HChG) Laser Beam in Plasma under Density Transition. Physics of Plasmas, 21, Article ID: 042101. http://dx.doi.org/10.1063/1.4870080

[20] Nanda, V., Kant, N. and Wani, M.A. (2013) Sensitiveness of Decentered Parameter for Relativistic Self-Focusing of a Hermite-Cosh-Gaussian Laser Beam in Plasma. IEEE Transactions on Plasma Science, 41, 2251-2256. http://dx.doi.org/10.1109/TPS.2013.2268164

[21] Kant, N., Wani, M.A. and Kumar, A. (2012) Self-Focusing of Hermite-Gaussian Laser Beams in Plasma under Plasma Density Ramp. Optics Communications, 285, 4483-4487. http://dx.doi.org/10.1016/j.optcom.2012.05.065

[22] Nanda, V., Kant, N. and Wani, M.A. (2013) Self-Focusing of a Hermite-Cosh Gaussian Laser Beam in a Magnetoplasma with Ramp Density Profile. Physics of Plasmas, 20, Article ID: 113109. http://dx.doi.org/10.1063/1.4833635 